\title{
Genetic testing for pulmonary stenosis
}

\author{
Yeltay Rakhmanov ${ }^{1}$, Paolo Enrico Maltese ${ }^{1 \star}$, Carla Marinelli ${ }^{1}$, Tommaso Beccari ${ }^{2}$, Munis Dundar ${ }^{3}$, \\ and Matteo Bertelli ${ }^{1,4}$
}

\begin{abstract}
Pulmonary stenosis (PS) is a congenital pulmonary valve malformation. It can be classified as valvular, subvalvular or supravalvular. Isolated forms of PS are rare. PS is associated with the development of massive pulmonary arterial dilatation. Patients with PS have a high consanguinity rate and the disorder is highly familial, which is why knowing the genetic aetiology of this defect is important. Prevalence is estimated at about 4/10,000 live births, and incidence at about $10 \%$ of all children with congenital heart defects. PS has prevalently autosomal dominant and rarely autosomal recessive-inheritance. This Utility Gene Test was developed on the basis of an analysis of the literature and existing diagnostic protocols. It is useful for confirming diagnosis, as well as for differential diagnosis, couple risk assessment and access to clinical trials.
\end{abstract}

Keywords: Pulmonary stenosis, EBTNA LAB UTILITY GENE TEST

${ }^{1}$ MAGI's Lab, Rovereto, Italy

${ }^{2}$ Department of Pharmaceutical Sciences, University of Perugia, Perugia, Italy

${ }^{3}$ Department of Medical Genetics, Erciyes University Medical School, Kayseri, Turkey

${ }^{4}$ MAGI Euregio, Bolzano, Italy

*Corresponding author: P. E. Maltese E-mail: paolo.maltese@assomagi.org

DOI: 10.2478/ebtj-2018-0039 (c) 2018 Authors. This work was licensed under the Creative Commons AttributionNonCommercial-NoDerivs 3.0 License.

\section{Genetic testing for pulmonary stenosis}

(Other synonyms: Pulmonic stenosis, pulmonary valve stenosis)

\section{General information about the disease}

Pulmonary stenosis (PS) is a congenital pulmonary valve malformation. Typical PS consists of a dome-shaped fusion of pulmonary semilunar valves, with poststenotic dilation of the main pulmonary artery, which is a common form of right ventricular outflow tract obstruction (1). Isolated PS is considered to be a rare congenital abnormality (2). PS is associated with the development of massive pulmonary arterial (PA) dilation. In a later period, this dilation may distort surrounding structures and compress the left main coronary artery or the left mainstem bronchus (3). Symptoms from such compression may mimic angina or asthma, respectively. In addition, significant PS may lead to symptoms of right heart failure including dyspnea, lower extremity edema, and chest pain (4).

Genetic factors are important because of the high consanguinity rate and high familial incidence, and the low frequency of irregularities during pregnancy (5).

Prevalence of PS is estimated at 4/10,000 live births, and incidence at about $10 \%$ of all children with congenital heart defects. About $10 \%$ of patients with PS have additional cardiac anomalies associated with Noonan (NS) and multiple lentigines/LEOPARD (ML/ LEOPARD) syndromes (1).

Diagnostic work-up includes clinical assessment to identify symptoms, echocardiogram, electrocardiogram, chest radiogram, integration of septal and color Doppler with twodimensional echocardiography, spin-echo MR imaging, CT, diagnostic catheterization and genetic testing.

Differential diagnosis should consider PS caused by chromosomal disorders and secondary PS. 
Inheritance is mainly autosomal dominant and rarely autosomal recessive. Sarkozy et al. reported non-syndromic PS (OMIM disease 265500) caused by PTPN11 (OMIM gene 176876) gene variations (6).

\section{Autosomal dominant syndromes associated with PS}

- Noonan syndrome 1 (NS1, OMIM disease 163950) PTPN11 (OMIM gene 176876);

- Noonan syndrome 8 (NS8, OMIM disease 615355) - RIT1 (OMIM gene 609591);

- Cardio-facio-cutaneous syndrome 1 (CFC1, OMIM disease 115150) - BRAF (OMIM gene 164757);

- Costello syndrome (CSTLO, OMIM disease 218040) - HRAS (OMIM gene 190020);

- Alagille syndrome 1 (ALGS1, OMIM disease 118450) - JAG1 (OMIM gene 601920);

- Alagille syndrome 2 (ALGS2, OMIM disease 610205) NOTCH2 (OMIM gene 600275);

- Noonan-like syndrome with loose anagen hair 1 (NSLH1, OMIM disease 607721) - SHOC2 (OMIM gene 602775);

- Mowat-Wilson syndrome (MOWS, OMIM disease 235730) - ZEB2 (OMIM gene 605802).

- Atrial septal defect 2 (ASD2, OMIM disease 607941) GATA4 (OMIM gene 600576);

- Ventricular septal defect 1 (VSD1, OMIM disease 614429) GATA4 (OMIM gene 600576);

- Supravalvar aortic stenosis (SVAS, OMIM disease 185500) ELN (OMIM gene 130160).

\section{Autosomal recessive syndrome associated with PS}

- Pitt-Hopkins-like syndrome-2 (PTHSL2, OMIM disease 614325) - NRXN1 (OMIM gene 600565)

Pathogenic variants may include missense, nonsense, small deletions, small indels, gross insertions and gross deletions.

\section{Aims of the test}

- To determine the gene defect responsible for the disease;

- To confirm clinical diagnosis;

- To assess the recurrence risk and perform genetic counselling for at-risk/affected individuals.

\section{Test characteristics}

\section{Specialist centers/ Published Guidelines}

The test is listed in the Orphanet database and is offered by 1 accredited medical genetic laboratories in the EU, and in the GTR database, offered by 6 accredited medical genetic laboratories in the US.

Guidelines for clinical use of the test are described in Genetics Home Reference (ghr.nlm.nih.gov).

\section{Test strategy}

A multi-gene next generation sequencing panel panel is used for the detection of nucleotide variations in coding exons and flanking introns of the above genes.
Potentially causative variants and regions with low coverage are Sanger-sequenced. Sanger sequencing is also used for family segregation studies.

Multiplex Ligation Probe Amplification (MLPA) is used to detect duplications and deletions in JAG1.

To perform molecular diagnosis, a single sample of biological material is normally sufficient. This may be $1 \mathrm{ml}$ peripheral blood in a sterile tube with $0.5 \mathrm{ml} \mathrm{K}$ EDTA or $1 \mathrm{ml}$ saliva in a sterile tube with $0.5 \mathrm{ml}$ ethanol $95 \%$. Sampling rarely has to be repeated. Gene-disease associations and the interpretation of genetic variants are rapidly developing fields. It is therefore possible that the genes mentioned in this note may change as new scientific data is acquired. It is also possible that genetic variants today defined as of "unknown or uncertain significance" may acquire clinical importance.

\section{Genetic test results \\ Positive}

Identification of pathogenic variants in the above genes confirms the clinical diagnosis and is an indication for family studies.

A pathogenic variant is known to be causative for a given genetic disorder based on previous reports, or predicted to be causative based on loss of protein function or expected significant damage to proteins or protein/protein interactions. In this way it is possible to obtain a molecular diagnosis in new/other subjects, establish the risk of recurrence in family members and plan preventive and/or therapeutic measures.

\section{Inconclusive}

Detection of a variant of unknown or uncertain significance (VUS): a new variation without any evident pathogenic significance or a known variation with insufficient evidence (or with conflicting evidence) to indicate it is likely benign or likely pathogenic for a given genetic disorder. In these cases, it is advisable to extend testing to the patient's relatives to assess variant segregation and clarify its contribution. In some cases, it could be necessary to perform further examinations/tests or to do a clinical reassessment of pathological signs.

\section{Negative}

The absence of variations in the genomic regions investigated does not exclude a clinical diagnosis but suggests the possibility of:

- alterations that cannot be identified by sequencing, such as large rearrangements that cause loss (deletion) or gain (duplication) of extended gene fragments;

- sequence variations in gene regions not investigated by this test, such as regulatory regions (5' and 3' UTR) and deep intronic regions;

- variations in other genes not investigated by the present test.

\section{Unexpected}

Unexpected results may emerge from the test, for example information regarding consanguinity, absence of family correlation or other genetically based-diseases. 


\section{Risk for progeny}

If the identified pathogenic variant has autosomal dominant transmission, the probability that an affected carrier transmit the disease variant to his/her children is $50 \%$ in any pregnancy, irrespective of the sex of the child conceived.

In autosomal recessive mutations, both parents are usually healthy carriers. In this case, the probability of transmitting the disorder to the offspring is $25 \%$ in any pregnancy of the couple, irrespective of the sex of the child. An affected individual generates healthy carrier sons and daughters in all cases, except in pregnancies with a healthy carrier partner. In these cases, the risk of an affected son or daughter is $50 \%$.

\section{Limits of the test}

The test is limited by current scientific knowledge regarding the gene and disease.

Analytical sensitivity (proportion of positive tests when the genotype is truly present) and specificity (proportion of negative tests when the genotype is not present)

NGS: Analytical sensitivity $>99.99 \%$, with a minimum coverage of 10X; Analytical specificity $99.99 \%$.

SANGER: Analytical sensitivity >99.99\%; Analytical specificit: 99.99\%.

MLPA: Analytical sensitivity $>99.99 \%$; Analytical specificity $99.99 \%$.

\section{Clinical sensitivity (proportion of positive tests if the disease is present) and clinical specificity (proportion of negative tests if the disease is not present)}

Clinical sensitivity: variations in the aforementioned genes are linked to PS, but may be individual variations (identified in one or a few families) and total epidemiological data is therefore not available.

Clinical specificity: is estimated at approximately 99\% (7)

\section{Prescription appropriateness}

The genetic test is appropriate when:

a) the patient meets the diagnostic criteria for PS;

b) the sensitivity of the test is greater than or equal to that of tests described in the literature.

\section{Clinical utility}

\begin{tabular}{l|c|}
\hline Clinical management & Utility \\
\hline Confirmation of clinical diagnosis & Yes \\
\hline Differential diagnosis & Yes \\
\hline Couple risk assessment & Yes \\
\hline $\begin{array}{l}\text { Availability of clinical trials can be checked on-line at } \\
\text { https://clinicaltrials.gov/ (8) }\end{array}$
\end{tabular}

\section{References}

1. Ferencz C, Loffredo CA, Correa-Villasenor A. Genetic and Environmental Risk Factors of Major Cardiovascular Malformations: The Baltimore-Washington Infant Study 1981-1989 Armonk, New York: Futura Publishing Co.

2. Blount SG, Komesu S, McCord MC. Asymptomatic isolated valvular pulmonary stenosis; diagnosis by clinical methods. N Engl J Med 1953; 248: 5-11

3. Hungate RG, Newman B, Meza MP. Left mainstem bronchial narrowing: a vascular compression syndrome? Evaluation by magnetic resonance imaging Pediatr Radiol 1998; 28(7): 527-32.

4. Morjaria S, Grinnan D, Voelkel N. Massive dilatation of the pulmonary artery in association with pulmonic stenosis and pulmonary hypertension. Pulmonary Circulation 2012; 2(2): 256-57.

5. Lamy M, De Grouchy J, Schweisguth O. Genetic and non-genetic factors in the etiology of congenital heart disease: a study of 1188 cases. Am J Hum Genet 1957; 9(1): 17-41.

6. Sarkozy A, Conti E, Esposito G, Pizzuti A, Dallapiccola B, Mingarelli R, Marino B, Digilio MC, Paoletti V. Nonsyndromic pulmonary valve stenosis and the PTPN11 gene. Am J Med Genet A 2003; 116A(4): 389-90.

7. B Chen, M Gagnon, S Shahangian, NL Anderson, DA Howerton, Boone JD; Centers for Disease Control and Prevention (CDC). Good Laboratory Practices for Molecular Genetic Testing for Heritable Diseases and Conditions. MMWR Recomm Rep 2009; 58 (RR-6): 1-37.

8. ClinicalTrials.gov ${ }^{\circledast}$ (Internet). U.S. National Library of Medicine. 